\title{
Publisher Correction: Making love in the time of corona - considering relationships in lockdown
}

\section{Marieke Dewitte, Chantelle Otten and Lauren Walker}

Correction to: Nature Reviews Urology 17, 547-553 (2020) https://doi.org/10.1038/s41585-020-0365-1, published online 20 August 2020.

The original HTML version of this article incorrectly attributed some text from one of the responses to Chantelle Otten instead of Lauren Walker. This error has now been corrected in the HTML version of the article.

https://doi.org/10.1038/s41585-020-00415-3 I Published online 14 December 2020

(c) Springer Nature Limited 2020 